\title{
Respuesta de la comunidad de fitoplancton y zooplancton al afloramiento de agua subterránea y surgencia costera en la península de Yucatán, México
}

\author{
Tomás I. Marina ${ }^{12,3,}$, ; Jorge A. Herrera-Silveira ${ }^{1}$ \& Israel Medina-GómeZ ${ }^{1}$ \\ ${ }^{1}$ Centro de Investigación y de Estudios Avanzados del Instituto Politécnico Nacional, Unidad Mérida, Mérida, México. ${ }^{2}$ \\ Consejo Nacional de Investigaciones Científicas y Técnicas (CONICET), Buenos Aires, Argentina. ${ }^{3}$ Instituto de Ciencias, \\ Universidad Nacional de General Sarmiento, Los Polvorines, Argentina.
}

\begin{abstract}
Resumen. Las descargas de agua subterránea (DAS) y la surgencia costera son fenómenos oceanográficos que generan nuevas condiciones hidroquímicas en áreas costeras. El cambio en la composición y la estructura de la comunidad planctónica ante estas condiciones determina la dinámica de los niveles tróficos bajos en los ecosistemas marino-costeros. El presente estudio describe la respuesta de la comunidad de fitoplancton y zooplancton al marco hidroquímico en dos ecosistemas costeros influenciados por dichos fenómenos en la península de Yucatán, una región tropical. Se realizaron tres campañas durante el año 2012 (junio, agosto y noviembre) y se muestrearon transectas de $10 \mathrm{~km}$ de extensión. Se midieron in situ temperatura, salinidad y oxígeno disuelto. Además, se colectaron muestras de agua para la determinación de nutrientes inorgánicos disueltos $\left(\mathrm{NO}_{2^{\prime}}, \mathrm{NO}_{3^{\prime}} \mathrm{NH}_{4}, \mathrm{PO}_{4}\right.$ y $\left.\mathrm{SiO}_{4}\right)$ y clorofila $a$, y análisis de componentes de la comunidad de fitoplancton

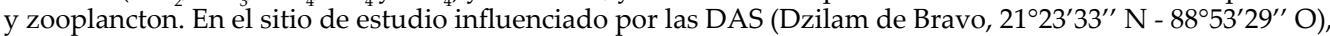
incrementos en la densidad de copépodos $(2484 \pm 890 \mathrm{org} / \mathrm{L})$, sincronizados con disminuciones en la densidad de diatomeas centrales, pennadas y dinoflagelados $(1600 \pm 1704,8730 \pm 8951$ y $22010 \pm 3557$ cél/L, respectivamente) en noviembre dan indicios de un cierto control de la comunidad de fitoplancton por parte de dicho grupo zooplanctónico durante momentos de mayor intensidad del fenómeno. Por otro lado, la surgencia costera de Cabo Catoche $\left(21^{\circ} 36^{\prime} 21^{\prime \prime} \mathrm{N}-87^{\circ} 06^{\prime} 12^{\prime \prime} \mathrm{O}\right)$ ocasionó un incremento en la densidad de diatomeas centrales ( $248000 \pm 126417$ cél/L), lo que resultó en una dominancia clara de este grupo durante el período de intensificación del fenómeno, mientras que los dinoflagelados dominaron en momentos de relajación de la surgencia. Las DAS en Dzilam de Bravo y la surgencia costera en Cabo Catoche generan respuestas diferenciales en la comunidad de plancton costero, e influyen en la estructura de esa comunidad y en la relación entre sus componentes.
\end{abstract}

[Palabras clave: estructura comunitaria, copépodos, diatomeas, dinoflagelados, Golfo de México, hidroquímica, plancton marino]

\begin{abstract}
Aвstract. Response of the phytoplankton and zooplankton community to groundwater discharge and oceanographic upwelling in the Yucatan Peninsula, México. Groundwater discharges (GWD) and coastal upwelling are oceanographic phenomena that generate new hydrochemical conditions in coastal areas. The change in the composition and structure of the phytoplankton and zooplankton community under these conditions determines the dynamics of low trophic levels in marine-coastal ecosystems. The present study describes the phytoplankton and zooplankton community response to the hydrochemical framework in two coastal ecosystems influenced by these phenomena in the Yucatan peninsula, a tropical region. For this purpose, three field campaigns were conducted during 2012 (June, August and November), sampling $10 \mathrm{~km}$ extension transects. Temperature, salinity and dissolved oxygen were measured in situ. In addition, water samples were collected for the determination of dissolved inorganic nutrients $\left(\mathrm{NO}_{2^{\prime}}, \mathrm{NO}_{3^{\prime}}, \mathrm{NH}_{4^{\prime}} \mathrm{PO}_{4}\right.$ and $\left.\mathrm{SiO}_{4}\right)$ and chlorophyll $a$, and analysis of components of the plankton community. At the study site influenced by GWD (Dzilam de Bravo, $\left.21^{\circ} 23^{\prime} 33^{\prime \prime} \mathrm{N}-88^{\circ} 53^{\prime} 29^{\prime \prime} \mathrm{O}\right)$, increases in Copepod density (2484 $\pm 890 \mathrm{org} / \mathrm{L}$ ) synchronized with considerable decreases in the density of centric and pennate diatoms and dinoflagellates $(1600 \pm 1704,8730 \pm 8951$ y $22010 \pm 3557 \mathrm{cel} / \mathrm{L}$, respectively) in November suggest that the phytoplankton community might be controlled by such zooplanktonic group during intensification of the discharges. On the other hand, the coastal upwelling off Cabo Catoche $\left(21^{\circ} 36^{\prime} 21^{\prime \prime} \mathrm{N}-87^{\circ} 06^{\prime} 12^{\prime \prime} \mathrm{W}\right)$ produced an increase in centric diatoms' density $(248000 \pm 126417$ $\mathrm{cel} / \mathrm{L})$, which resulted in a clear dominance of this group in the period of intensification of the phenomenon, whereby dinoflagellates dominate during relaxation of the upwelling. GWD in Dzilam de Bravo and coastal upwelling off Cabo Catoche generate differential responses in the community of coastal plankton, influencing the structure of such community and the relationships between its components.
\end{abstract}

[Keywords: community structure, copepods, diatoms, dinoflagellates, Gulf of México, hydrochemistry, marine plankton]

Editora asociada: Irina Izaguirre

tomasimarina@gmail.com 


\section{INTRODUCCIÓN}

La península de Yucatán (PY), en el sureste de México, es una formación cárstica limitada por el Mar Caribe y el Golfo de México. Los estudios hidroquímicos en la región mostraron que el principal aporte de nutrientes en la costa está vinculado a dos fenómenos de naturaleza estacional: uno, de índole hidrometeorológica en las descargas de agua subterránea (DAS) en el centro-norte de la PY (Kantun-Manzano 2011; Enríquez et al. 2013); el segundo, oceanográfico, materializado en la surgencia costera que ocurre en el centro-este de la PY (Merino 1992, 1997). Mientras que la intensidad de las DAS tiene un carácter ligado al patrón de la precipitación y a la recarga del acuífero (Herrera-Silveira 1993), la surgencia costera exhibe un comportamiento dinámico asociado tanto con la corriente de Yucatán como con los vientos locales (Enríquez and Mariño-Tapia 2014; Reyes-Mendoza et al. 2015).

En el norte de la PY, las DAS se caracterizan por presentar concentraciones elevadas de nitratos y silicatos $(6.1$ y $6.61 \mu \mathrm{M}$, respectivamente) (Troccoli-Ghinaglia et al. 2010), con descargas máximas que alcanzan los $40000 \mathrm{~m}^{3}$ /día (Valle-Levinson et al. 2011). Por otro lado, la surgencia costera aflora de una profundidad cercana a los $200 \mathrm{~m}$ del canal de Yucatán, y consiste en agua rica en nitratos, fosfatos y silicatos (8-14, 1.2-1.7 y 4.5-7.3 $\mu \mathrm{M}$, respectivamente) (Merino 1997; Reyes-Mendoza 2010).

Diversos estudios sobre la influencia de las DAS y las surgencias costeras en el fitoplancton y zooplancton han sido realizados en regiones templadas y subtropicales (Johannes 1980; Estrada and Blasco 1985; Gobler and Boniello 2003; Peterson et al. 2007; Huang et al. 2011). Sin embargo, las investigaciones dirigidas a conocer el impacto de estos fenómenos sobre la dinámica del plancton en ecosistemas marino-costeros tropicales no han sido tan preponderantes como en latitudes superiores $\mathrm{y}$, por lo tanto, el entendimiento sobre esta interrelación es todavía parcial y localizado en algunos sitios del Caribe (Corredor 1979; Jiménez 1981; Álvarez-Góngora and HerreraSilveira 2006; Troccoli-Ghinaglia et al. 2010).

Es sabido que cambios en la concentración de nutrientes pueden alterar la composición y estructura de los productores primarios en ecosistemas marinos (Stolte et al. 1994; Legendre and Rassoulzadegan 1995; Cloern et al. 2014). Dicho mecanismo opera en las comunidades planctónicas mediante un control bottom-up, a nivel del balance entre crecimiento y pastoreo, y se refleja en la producción primaria y secundaria (Margalef 1967). En consecuencia, oscilaciones en la disponibilidad de nutrientes tienen el potencial de influenciar a escala regional procesos ecológicamente significativos como pesquerías y ciclo del carbono.

Los estudios realizados sobre la comunidad fitoplanctónica en la región costera de la PY reportan una dominancia de diatomeas pennadas y dinoflagelados, principalmente especies bentónicas (Troccoli-Ghinaglia et al. 2004; 2010; Álvarez-Góngora et al. 2012). Esos estudios sugieren que la predominancia del componente bentónico dentro de la comunidad es consecuencia de la resuspensión en la columna de agua promovida por el flujo vertical ascendente del efluente de las DAS, compensado por corrientes asimétricas descendentes en las inmediaciones del manantial (Valle-Levinson et al. 2011). Por otro lado, la relevancia ecológica del evento de surgencia costera en la PY en los niveles tróficos bajos ha sido poco explorada, y sólo existen investigaciones sobre el efecto del fenómeno en especies bandera con atributos carismáticos para el desarrollo turístico de la región como el tiburón ballena y la manta raya (Hinojosa-Álvarez et al. 2012; CárdenasPalomo et al. 2015).

La hipótesis de disturbio intermedio afirma que la biodiversidad dentro de una comunidad está relacionada con la frecuencia e intensidad de cualquier disturbio y plantea que los mayores valores de diversidad se registran a niveles intermedios de frecuencia o intensidad (Connell 1978). En el plancton se analizó el efecto de los disturbios y se encontró que a mayor frecuencia y/o intensidad de los mismos suele registrarse una diversidad menor (Padisák et al. 1993). Las pocas especies que sobrevivan o se adapten a las nuevas condiciones generadas por el disturbio serán las dominantes. En este contexto, procesos de conectividad tierra-mar como las DAS y océano-mar como la surgencia costera podrían ser considerados disturbios intermedios para la comunidad de plancton ya que originan nuevas condiciones hidroquímicas locales (i.e., incremento en la concentración de nutrientes) que podrían maximizar la diversidad en dicha comunidad (Reynolds 1993; Flöder and Sommer 1999). Los estudios orientados a entender la relación entre dicha comunidad y la matriz ambiental son relevantes por su importancia en las tramas tróficas de los 
ecosistemas marinos (Odum and Warrett 2006). El interés general de investigar el comportamiento variable de dichas interacciones emana del papel fundamental que ejercen sobre los ciclos biogeoquímicos en el ambiente costero; los mismos pueden verse alterados por procesos que actúan a mayor escala fuera del nivel espacial de medición (e.g., actividades antrópicas, cambios en los movimientos de las masas de agua, eventos climatológicos inter-anuales: ENSO).

Los objetivos del presente estudio son: a) caracterizar hidroquímicamente la variación en dos sitios de estudio, uno influenciado por las DAS (Dzilam de Bravo) y otro por la surgencia costera (Cabo Catoche), y b) describir y comparar la respuesta de la comunidad de fitoplancton y zooplancton durante períodos de intensificación de dichos fenómenos oceanográficos. Nuestra hipótesis de trabajo sostiene que la respuesta de la comunidad de fitoplancton en ambos sitios estará dominada por un control bottom-up como consecuencia de la importante entrada de nutrientes durante la intensificación de los fenómenos.

\section{Materiales y Métodos}

\section{Área de estudio}

El estudio se llevó a cabo en el norte de la zona costera de la PY, sureste del Golfo de México. El clima es seco y árido, con una estación lluviosa en el verano y escasas lluvias durante el resto del año. Existen tres estaciones bien marcadas: seca (marzo-mayo), lluviosa (junio-octubre) y de vientos fríos o "nortes" (noviembre-febrero).

El estudio se realizó en dos sitios de la península: Dzilam de Bravo y Cabo Catoche (Figura 1). Dzilam de Bravo se encuentra en la costa centro-norte. Sus aguas costeras tienen entre 2 y $8.7 \mathrm{~m}$ de profundidad y el fondo está cubierto de pastos marinos y macroalgas (Herrera-Silveira et al. 2004). La zona costera se caracteriza por afloramientos de agua dulce, "ojos de agua dulce", que son DAS puntual o difusa con altas concentraciones de nitratos y silicatos (Herrera-Silveria 2006). Por otro lado, Cabo Catoche se encuentra en la punta más septentrional de la PY. La región del cabo, cuya profundidad varía entre 3.3 y $14 \mathrm{~m}$, está influenciada por la corriente de Yucatán, que fluye muy cerca de la costa y domina las características oceanográficas de la región. Esta corriente proporciona la mayor parte del flujo al Golfo de México (Ochoa et al. 2001). La presencia de un evento de surgencia costera estacional, en el periodo marzo-septiembre, está bien documentado (Merino 1997; ReyesMendoza et al. 2015).

\section{Muestreos y análisis de laboratorio}

Se realizaron muestreos en los meses de junio, agosto y noviembre de 2012 en los dos sitios de estudio; un muestreo por mes y sitio. En cada sitio se estableció una transecta de $10 \mathrm{~km}$ perpendicular a la línea de costa con el objetivo de captar la variación espacial costa-mar. A lo largo de dicha transecta se distribuyeron 10 estaciones de muestreo equidistantes 1 $\mathrm{km}$, y se estableció la más próxima a la costa a $1 \mathrm{~km}$ de la línea intermareal (Figuras 2 y 3 , estación 1). En cada estación se registraron in situ datos de temperatura superficial $\left({ }^{\circ} \mathrm{C}\right)$, salinidad (ups) y oxígeno disuelto $(\mathrm{mg} / \mathrm{L})$ con una sonda multiparamétrica YSI 85 (Yellow Spring Incorporated, Ohio, USA). Además, se tomó una muestra de agua superficial $(\sim 1 \mathrm{~m}$ de profundidad) en cada estación, se almacenaron en botellas de plástico tipo Nalgene de $1 \mathrm{~L}$ y se colocaron a $4{ }^{\circ} \mathrm{C}$ en una conservadora térmica para su posterior análisis de concentración

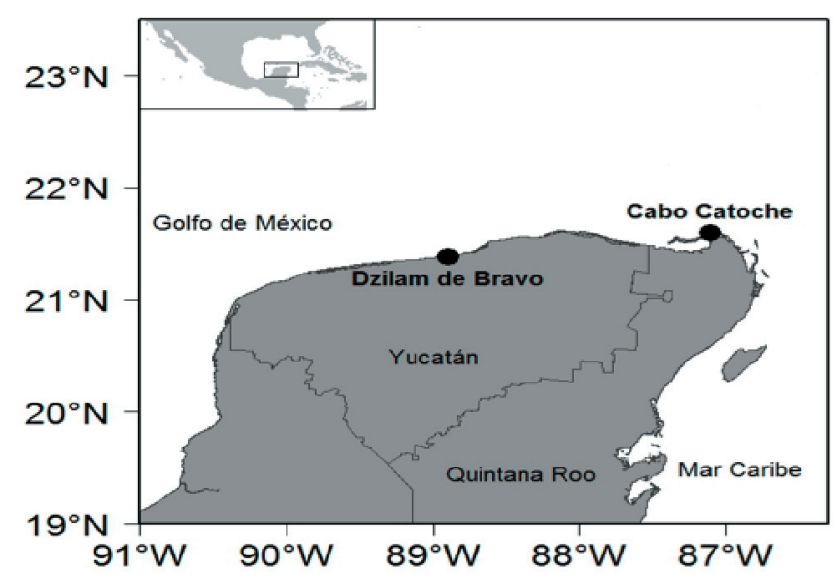

Figura 1. Mapa de la península de Yucatán donde se localizan los sitios de estudio: Dzilam de Bravo y Cabo Catoche.

Figure 1. Map of the Yucatan Peninsula where the study sites are shown: Dzilam de Bravo and Cabo Catoche. 
a)

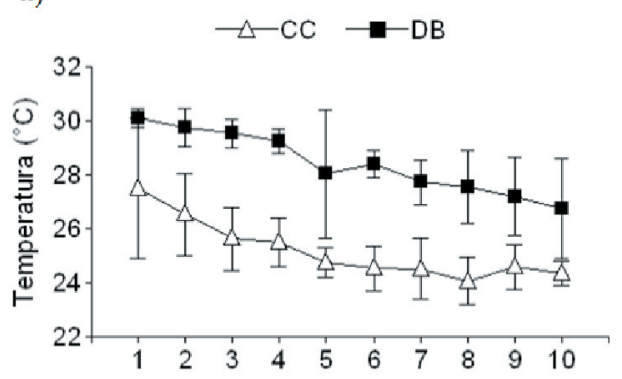

c)

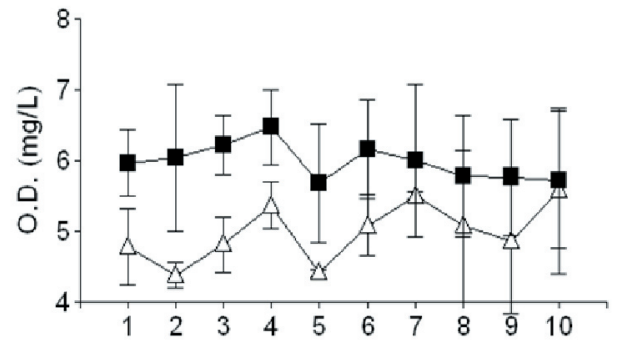

e)

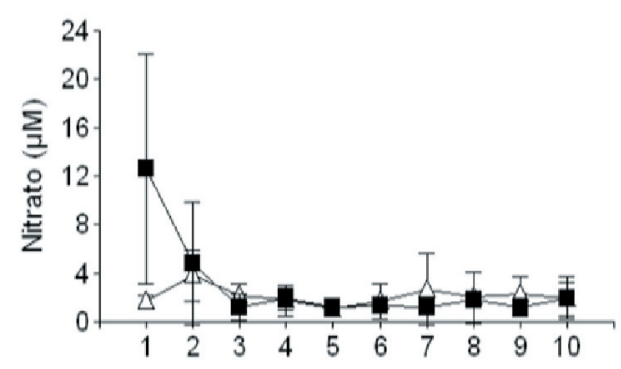

g)

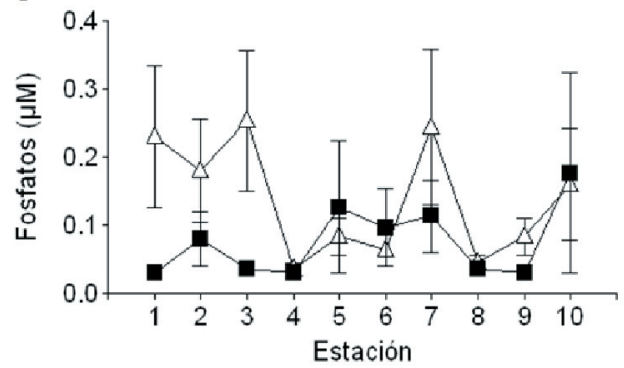

b)

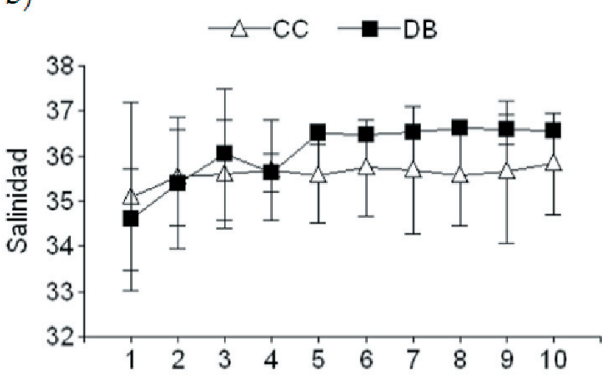

d)

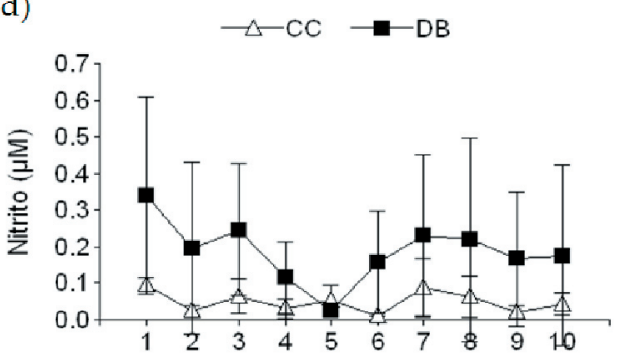

f)

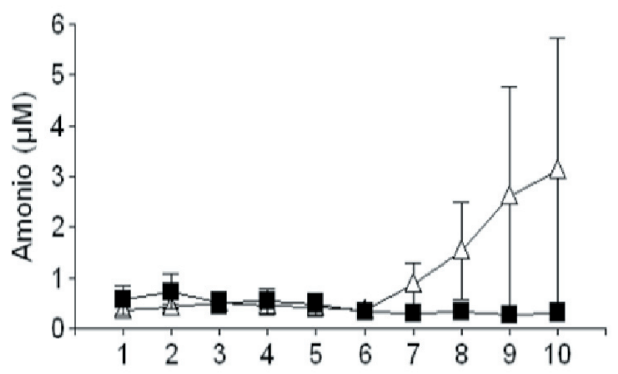

h)

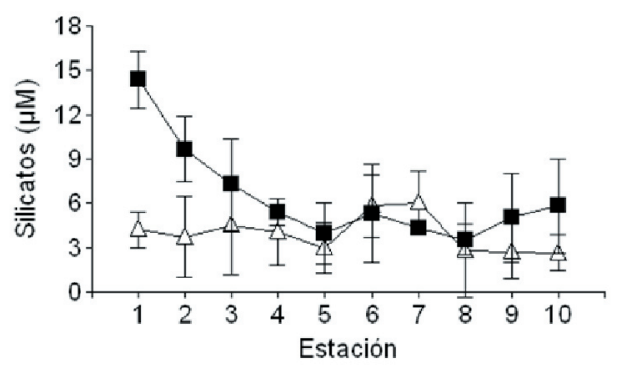

Figura 2. Variación espacial (media \pm desviación estándar) de: a) temperatura; b) salinidad; y concentraciones de c) oxígeno disuelto (O.D.); d) nitritos; e) nitratos; f) amonio; g) fosfatos; h) silicatos. DB: Dzilam de Bravo, CC: Cabo Catoche. Se consideraron los tres muestreos realizados (junio, agosto y noviembre). 1 a 10 corresponden a las estaciones de muestreo, estación 1: más cercana a la costa.

Figure 2. Spatial variation (mean \pm standard deviation) of: $a$ ) temperature; $b$ ) salinity; and concentrations of c) dissolved oxygen (O.D.); d) nitrates; e) nitrites; f) ammonium; g) phosphates; h) silicates. DB: Dzilam de Bravo, CC: Cabo Catoche. Shown data consider the three sampling campaigns (June, August and November). 1 to 10 corresponds to sampling stations, station 1: nearest to the coast.

de nutrientes y clorofila $a$ (cl-a). Asimismo, en cada estación se tomaron $250 \mathrm{~mL}$ de agua superficial para el análisis cualitativo y cuantitativo de fitoplancton y se les agregaron $2.5 \mathrm{~mL}$ de Lugol neutralizado con acetato de sodio para su preservación. Las muestras de zooplancton se obtuvieron mediante arrastres horizontales superficiales de cinco minutos con una red tipo cono truncado $(0.4 \mathrm{~m}$ de boca, 1.4 de longitud y $300 \mu \mathrm{m}$ de malla) realizados en cada estación. Este tipo de red ha sido amplia y exitosamente utilizada para el estudio de la comunidad de zooplancton en la PY (SuárezMorales and Gasca 2000; Ordoñez-López 
a)

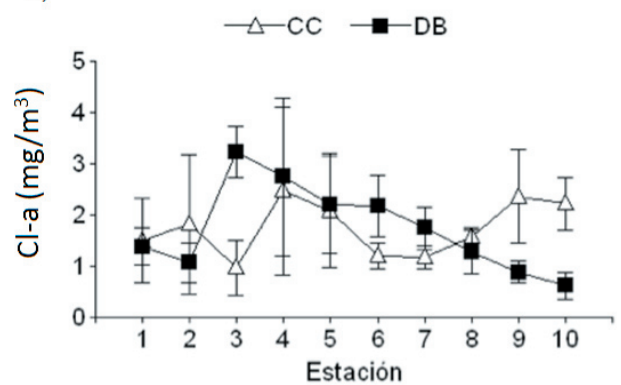

b)

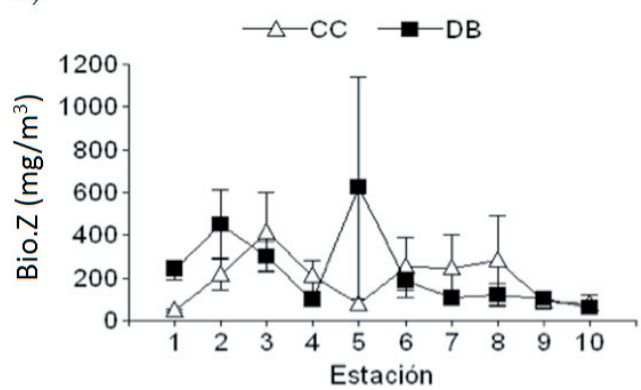

Figura 3. Variación espacial (media desviación estándar) de: a) concentración cl-a; b) biomasa de zooplancton (Bio.Z). DB: Dzilam de Bravo, CC: Cabo Catoche. Se consideraron los tres muestreos realizados (junio, agosto y noviembre).

Figure 3. Spatial variation (mean \pm standard deviation) of: a) cl-a concentration; b) zooplankton biomass (Bio.Z). DB: Dzilam de Bravo, CC: Cabo Catoche. Shown data consider the three sampling campaigns (june, august and november).

and Ornelas-Roa 2003; Álvarez-Cadena et al. 2007). Dichas muestras (250 mL), utilizadas para las estimaciones de densidad y biomasa, fueron fijadas en el campo con formaldehído en agua de mar neutralizado con borato de sodio a $\mathrm{pH}=7.5-8.0$. Todas las muestras fueron colectadas entre las 9:00 am y la 1:00 pm, hora local.

Las concentraciones de nutrientes (nitritos, nitratos, amonio, fosfatos y silicatos) y cla se analizaron en laboratorio por medio de técnicas espectrofotométricas estándar descritas por Parsons et al. (1984). Para el análisis de cl-a el agua fue filtrada a través de filtros de membrana Millipore de $25 \mathrm{~mm}$ de diámetro y $0.45 \mu \mathrm{m}$ de poro, realizándose la extracción con acetona al 90\% y calculándose su concentración de acuerdo a las ecuaciones descritas por Jeffrey y Humphrey (1975).

Los análisis de la comunidad de fitoplancton y zooplancton se realizaron en el Laboratorio de Plancton del CINVESTAV-IPN. El fitoplancton se analizó siguiendo la técnica de Utermöhl, referida por Hasle (1978): las muestras se sedimentaron por $24 \mathrm{~h}$ en cámaras de Utermöhl de $50 \mathrm{~mL}$ y se observaron en un microscopio invertido Axiovert 100 (Zeiss, Germany) con el objetivo 10x (NA=0.25). Se contaron 100 campos con coordenadas al azar. La identificación taxonómica se realizó de acuerdo a las referencias de Sournia (1986) y Round et al. (1990), clasificando a los organismos en los siguientes grandes grupos: diatomeas centrales, diatomeas pennadas y dinoflagelados. Se consideraron estos grupos por ser los más abundantes en la PY (TroccoliGhinaglia et al. 2004; 2010; Álvarez-Góngora et al. 2012). También se obtuvieron datos de densidad (cél/L) de cada grupo fitoplanctónico. El zooplancton se analizó con base en alícuotas de $10 \mathrm{~mL}$ y observación en un microscopio estereoscópico. La biomasa zooplanctónica $\left(\mathrm{mg} / \mathrm{m}^{3}\right)$ fue cuantificada por el método de peso húmedo (Omori and Ikeda 1984), y la abundancia se estandarizó a organismos por litro. La identificación taxonómica en grandes grupos se llevó a cabo de acuerdo a las referencias de Boltovskoy (1999).

El análisis de la información consistió en determinar posibles diferencias significativas de las variables hidroquímicas y planctónicas (densidad y biomasa de grupos analizados) entre los meses muestreados en cada sitio a través deun análisis devarianza noparamétrico utilizando la prueba de significancia de Kruskal-Wallis $(P<0.05)$ mediante el software Infostat (Di Rienzo et al. 2014). Además, se compararon los meses de mayor intensidad de los fenómenos (Kruskal-Wallis, $P<0.1$ ). La asociación de los grupos de plancton definidos con las variables hidroquímicas se estudió mediante un análisis multivariado: Análisis de Correspondencia Canónica (ACC), el cual permite posicionar a los taxa con los factores ambientales en un sistema sintético de coordenadas (ter Braak and Verdonschot 1995). Para esto, se utilizó la densidad de cada grupo planctónico (diatomeas centrales, pennadas, dinoflagelados y copépodos (grupo zooplanctónico más abundante) y las variables hidroquímicas temperatura superficial, salinidad, oxígeno disuelto (OD), concentraciones de amonio $\left(\mathrm{NH}_{4}\right)$, nitratos $\left(\mathrm{NO}_{3}\right)$, fosfatos $\left(\mathrm{PO}_{4}\right)$, silicatos $\left(\mathrm{SiO}_{4}\right)$ y cl-a. Se utilizaron los datos de los tres meses para cada sitio en el ACC, el cual se realizó con el paquete vegan del software libre $R$, versión 0.98 (Oksanen et al. 2013).

\section{Resultados}

El análisis de las variables hidroquímicas y planctónicas permitió identificar diferencias 
Tabla 1. Resumen de las variables hidroquímicas y planctónicas (media \pm desviación estándar) en Dzilam de Bravo y Cabo Catoche. Diferentes letras indican diferencias significativas en cada sitio (Kruskal-Wallis, $P<0.05$ ). Temp: temperatura, Sal.: salinidad, O.D.: oxígeno disuelto. s/d: sin datos.

Table 1. Summary of the hydrochemical and planktonic variables (mean \pm standard deviation) in Dzilam de Bravo and Cabo Catoche. Different letters indicate significant differences in each site (Kruskal-Wallis, $P<0.05)$. Temp: temperature, Sal.: salinity, O.D.: dissolved oxygen. s/d: no data.

\begin{tabular}{|c|c|c|c|c|c|c|}
\hline \multirow[b]{2}{*}{ Mes } & \multicolumn{3}{|c|}{ Dzilam de Bravo } & \multicolumn{3}{|c|}{ Cabo Catoche } \\
\hline & junio & agosto & noviembre & junio & agosto & noviembre \\
\hline \multicolumn{7}{|l|}{ Variables hidroquímicas } \\
\hline $\operatorname{Temp}\left({ }^{\circ} \mathrm{C}\right)$ & $28.1 \pm 1.7$ & $28.1 \pm 1.9$ & $29.1 \pm 0.4$ & $25.7 \pm 1.4$ & $25 \pm 2.1$ & $24.9 \pm 0.2$ \\
\hline Sal. (ups) & $36.4 \pm 0.5$ & $36.2 \pm 0.7$ & $35.7 \pm 1.3$ & $34.2 \pm 0.6^{\mathrm{a}}$ & $36.5 \pm 0.3^{b}$ & $36.1 \pm 0.3^{b}$ \\
\hline O.D. (mg/L) & $6.8 \pm 0.20^{\mathrm{b}}$ & $5.5 \pm 0.40^{\mathrm{a}}$ & $5.6 \pm 0.40^{\mathrm{a}}$ & $4.7 \pm 0.03$ & $\mathrm{~s} / \mathrm{d}$ & $5.2 \pm 0.70$ \\
\hline $\mathrm{NO}_{2}(\mu \mathrm{M})$ & $0.03 \pm 0.02^{\mathrm{a}}$ & $0.30 \pm 0.20^{\mathrm{b}}$ & $0.23 \pm 0.20^{\mathrm{b}}$ & $0.04 \pm 0.03$ & $0.07 \pm 0.05$ & $0.03 \pm 0.04$ \\
\hline $\mathrm{NO}_{3}(\mu \mathrm{M})$ & $1.58 \pm 0.5$ & $3.06 \pm 3.8$ & $4.11 \pm 6.9$ & $1.55 \pm 0.3^{\mathrm{ab}}$ & $3.30 \pm 1.4^{\mathrm{b}}$ & $1.38 \pm 1.7^{\mathrm{a}}$ \\
\hline $\mathrm{NH}_{4}(\mu \mathrm{M})$ & $0.25 \pm 0.2$ & $0.24 \pm 0.2$ & $0.28 \pm 0.3$ & $0.40 \pm 0.5$ & $2.18 \pm 3.3$ & $0.25 \pm 0.2$ \\
\hline $\mathrm{PO}_{4}(\mu \mathrm{M})$ & $0.04 \pm 0.02$ & $0.11 \pm 0.1$ & $0.08 \pm 0.1$ & $0.12 \pm 0.1$ & $0.19 \pm 0.1$ & $0.11 \pm 0.1$ \\
\hline $\mathrm{SiO}_{4}(\mu \mathrm{M})$ & $7.5 \pm 3.3$ & $6.4 \pm 3.8$ & $5.5 \pm 4.3$ & $4.8 \pm 2.3^{\mathrm{b}}$ & $1.9 \pm 1.5^{\mathrm{a}}$ & $5.1 \pm 1.3^{\mathrm{b}}$ \\
\hline $\mathrm{N}: \mathrm{P}$ & $55 \pm 25$ & $92 \pm 138$ & $124 \pm 234$ & $31 \pm 18$ & $66 \pm 82$ & $21 \pm 14$ \\
\hline \multicolumn{7}{|l|}{ Variables planctónicas } \\
\hline $\mathrm{Cl}-\mathrm{a}\left(\mathrm{mg} / \mathrm{m}^{3}\right)$ & $1.08 \pm 0.7$ & $2.43 \pm 1.8$ & $1.7 \pm 0.6$ & $1.24 \pm 1.4^{\mathrm{a}}$ & $2.80 \pm 1.5^{\mathrm{a}}$ & $1.17 \pm 0.3^{\mathrm{b}}$ \\
\hline Biomasa zooplancton $\left(\mathrm{mg} / \mathrm{m}^{3}\right)$ & $321.68 \pm 485.9$ & $150.3 \pm 104.5$ & $210.02 \pm 218.3$ & $292.8 \pm 215.8^{\mathrm{b}}$ & $238.77 \pm 218.5^{\mathrm{b}}$ & $43.22 \pm 23.3^{\mathrm{a}}$ \\
\hline Diatomeas centrales (cél/L) & $8310 \pm 7890^{\mathrm{b}}$ & $13750 \pm 13096^{\mathrm{b}}$ & $1600 \pm 1704^{\mathrm{a}}$ & $560 \pm 818^{\mathrm{a}}$ & $248000 \pm 126417^{b}$ & $1780 \pm 1897^{\mathrm{a}}$ \\
\hline Diatomeas pennadas (cél/L) & $12980 \pm 7852$ & $11740 \pm 7769$ & $8730 \pm 8951$ & $4830 \pm 6241$ & $2850 \pm 4180$ & $1310 \pm 493$ \\
\hline Dinoflagelados (cél/L) & $31280 \pm 11540$ & $26100 \pm 14424$ & $22010 \pm 3557$ & $132910 \pm 144776^{\mathrm{b}}$ & $13710 \pm 5591^{a}$ & $15430 \pm 3944^{\mathrm{a}}$ \\
\hline Copépodos (org/L) & $1107 \pm 882^{a}$ & $1643 \pm 1300^{\mathrm{ab}}$ & $2484 \pm 890^{\mathrm{b}}$ & $1558 \pm 809^{\mathrm{b}}$ & $848 \pm 512^{\mathrm{ab}}$ & $627 \pm 199^{a}$ \\
\hline
\end{tabular}

Tabla 2. Comparación de las variables hidroquímicas y planctónicas durante los períodos de intensificación de las DAS (noviembre, Dzilam de Bravo) y la surgencia costera (agosto, Cabo Catoche). Sólo se muestran las variables significativamente diferentes. Prueba no paramétrica (Kruskal-Wallis). ${ }^{*}<0.1,{ }^{* *}<0.05,{ }^{* * *}<0.01,{ }^{* * * *}<0.001 . \mathrm{n}=10$ (corresponde a las diez estaciones de la transecta).

Table 2. Comparison of the hydrochemical and planktonic variables during periods of intensification of SWD (November, Dzilam de Bravo) and coastal upwelling (August, Cabo Catoche). Only the variables that showed significant differences are presented. Non-parametric test (Kruskal-Wallis). ${ }^{*}<0.1,{ }^{* *}<0.05,{ }^{* * *}<0.01,{ }^{* * * *}<0.001 . \mathrm{n}=10$ (it corresponds to the ten stations of the transect).

\begin{tabular}{cc}
\hline Variables & DAS vs surg. cost. \\
\hline Hidroquímicas & $* * *$ \\
Temperatura & $* *$ \\
$\mathrm{NO}_{2}$ & $* *$ \\
$\mathrm{NO}_{3}$ & $*$ \\
$\mathrm{NH}_{4}$ & $*$ \\
$\mathrm{PO}_{4}$ & $* *$ \\
$\mathrm{SiO}_{4}$ & $* *$ \\
Planctónicas $\mathrm{Cl}^{-a}$ & $* * * *$ \\
Centrales & $* *$ \\
Pennadas & $* * *$ \\
Dinoflagelados & $* * *$ \\
Copépodos
\end{tabular}

temporales (entre meses) y espaciales (costamar) en Dzilam de Bravo y Cabo Catoche, sitios influenciados por las DAS y la surgencia costera, respectivamente (Tabla 1). Por otro lado, durante la fecha de muestreo de mayor intensidad de los fenómenos, noviembre para las DAS y agosto en el caso de la surgencia costera, también se observaron diferencias en las variables entre los sitios (Tabla 2).

\section{Dzilam de Bravo: influencia de las DAS}

En Dzilam de Bravo, la temperatura y la salinidad no registraron diferencias temporales significativas, aunque la mayor temperatura y la menor salinidad medias se presentaron en noviembre. Las concentraciones de nitratos $\left(\mathrm{NO}_{3}\right)$ y amonio $\left(\mathrm{NH}_{4}\right)$ fueron más elevadas en noviembre, mientras que los silicatos $\left(\mathrm{SiO}_{4}\right)$ presentaron valores relativamente más bajos en ese mes. Como consecuencia de menores concentraciones de fosfatos con respecto a los compuestos nitrogenados, la relación Redfield $(\mathrm{N}: \mathrm{P})$ fue mucho mayor que el valor teórico de 16:1 en todos los meses, y mostró su valor máximo en noviembre (Tabla 1). En cuanto a la cl-a, las concentraciones más elevadas se registraron en agosto y noviembre (2.43 y 1.70 $\mathrm{mg} / \mathrm{m}^{3}$, respectivamente). Espacialmente, la temperatura, el oxígeno disuelto (O.D.), la concentración de nutrientes (a excepción de los fosfatos $-\mathrm{PO}_{4}^{-}$) y la cl-a presentaron mayores valores en las estaciones próximas a la costa (Figuras 2a-h y 3a, estaciones 1 a 5).

Los grupos fitoplanctónicos estudiados, diatomeas centrales y pennadas y dinoflagelados, registraron las menores densidades en noviembre. Es importante notar que en dicho mes las diatomeas 
centrales presentaron una densidad significativamente menor al resto de los meses (Tabla 1). Por el contrario, la densidad de copépodos fue significativamente mayor en noviembre, y mostró una relación opuesta con las diatomeas centrales. Las mayores biomasas del zooplancton se registraron en noviembre y junio $\left(210.02\right.$ y $321.68 \mathrm{mg} / \mathrm{m}^{3}$, respectivamente). Espacialmente, las primeras estaciones de la transecta presentaron las biomasas zooplanctónicas más altas (Figura $3 \mathrm{~b}$, estaciones 1 a 5 ).

En las muestras de zooplancton se identificaron 16 grupos taxonómicos durante los tres muestreos. El dominante fue el de los copépodos (91.9\%). El 8.1\% restante se repartió entre los taxa Lamellibranchia (Bivalvia), Amphipoda, Echinodermata, Appendicularia, Polychaeta (larva trocophora) y Decapoda (etapa larval zoea) (Figura 4a).

El análisis multivariado (ACC) mostró asociación de los grupos de fitoplancton y los copépodos con las variables hidroquímicas. En el eje canónico I (eigenvalue: 0.1086, varianza explicada: $73.12 \%$ ) las diatomeas centrales se asociaron con la cl-a, fosfatos y salinidad, mientras que las diatomeas pennadas lo hicieron con los nitratos, amonio y silicatos. Al eje II (eigenvalue: 0.0297, varianza explicada: $19.99 \%$ ) se asociaron los dinoflagelados y copépodos con el oxígeno disuelto (O.D.) y la temperatura (Figura 5a).

\section{Cabo Catoche: influencia de la surgencia costera}

En Cabo Catoche, la temperatura superficial no mostró variaciones temporales importantes $\left(<1^{\circ} \mathrm{C}\right)$ mientras que la salinidad sí presentó

a)

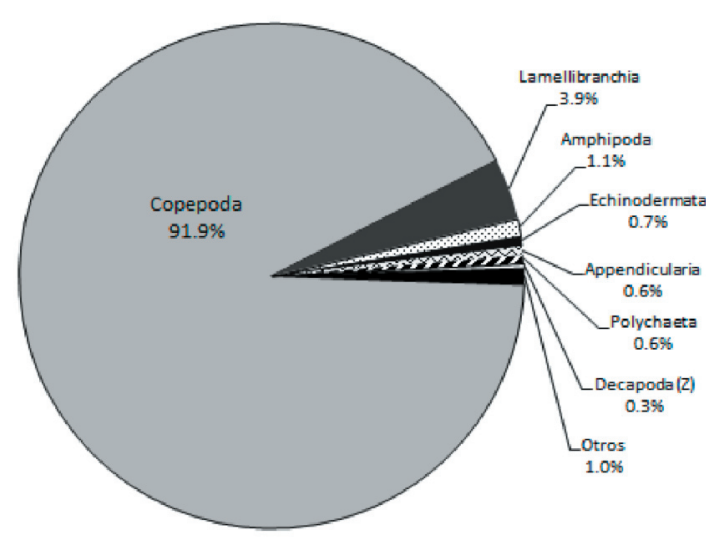

diferencias significativas $(P<0.05)$, y fue mayor en agosto. La concentración media de todos los nutrientes, con excepción de los silicatos $\left(\mathrm{SiO}_{4}\right)$, presentó el mismo comportamiento temporal; los mayores valores se registraron en agosto, y los menores, en noviembre (Tabla 1). Contrariamente, los silicatos presentaron la concentración media más alta en noviembre y la más baja en agosto (5.06 y $1.94 \mu \mathrm{M}$, respectivamente). Al igual que en Dzilam de Bravo, la relación Redfield (N:P) fue mucho mayor que el valor teórico de 16:1 en todos los meses, y mostró su valor máximo en agosto. La cl-a fue significativamente mayor en agosto $\left(2.8 \mathrm{mg} / \mathrm{m}^{3}\right)$ (Tabla 1). Espacialmente, la temperatura y la salinidad se comportaron de manera opuesta, con temperaturas más bajas y salinidades más elevadas en estaciones más alejadas de la costa (Figura 2a-b, estaciones 6 a 10). En cuanto a los nutrientes, sólo se observó un patrón en la concentración de amonio que aumenta en zonas alejadas de la costa, al igual que la cl-a (Figuras 2c-h y 3a, estaciones 6 a 10).

En cuanto a la densidad de los grupos fitoplanctónicos, las diatomeas centrales registraron diferencias significativas (KruskalWallis, $P<0.05)$ con la mayor densidad en agosto, mientras que los dinoflagelados registraron la mayor densidad en junio, significativamente diferente al resto de los meses (Tabla 1). Las diatomeas pennadas también presentaron la mayor densidad en junio (4830 cél/L). La densidad de copépodos fue significativamente mayor en junio al igual que su biomasa (Tabla 1). Espacialmente, las biomasas variaron a lo largo de la transecta (Figura 3b).

b)

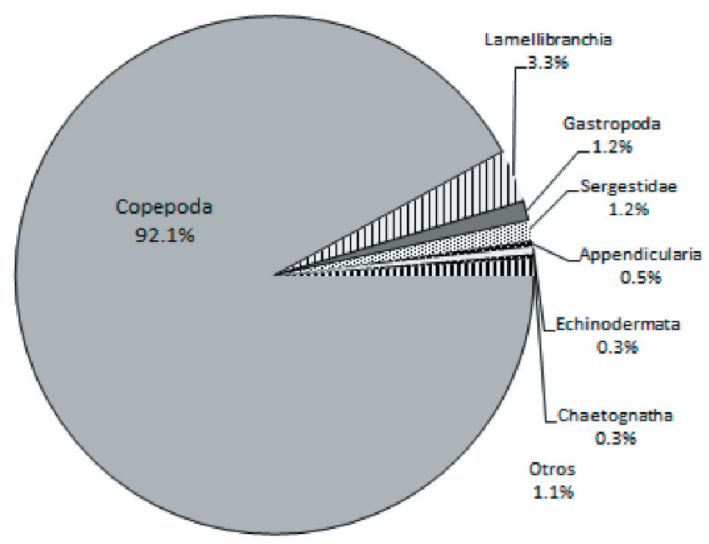

Figura 4. Principales grupos taxonómicos de zooplancton identificados en a) Dzilam de Bravo y b) Cabo Catoche.

Figure 4. Principal taxonomic groups of zooplankton identified in a) Dzilam de Bravo and b) Cabo Catoche. 
a)

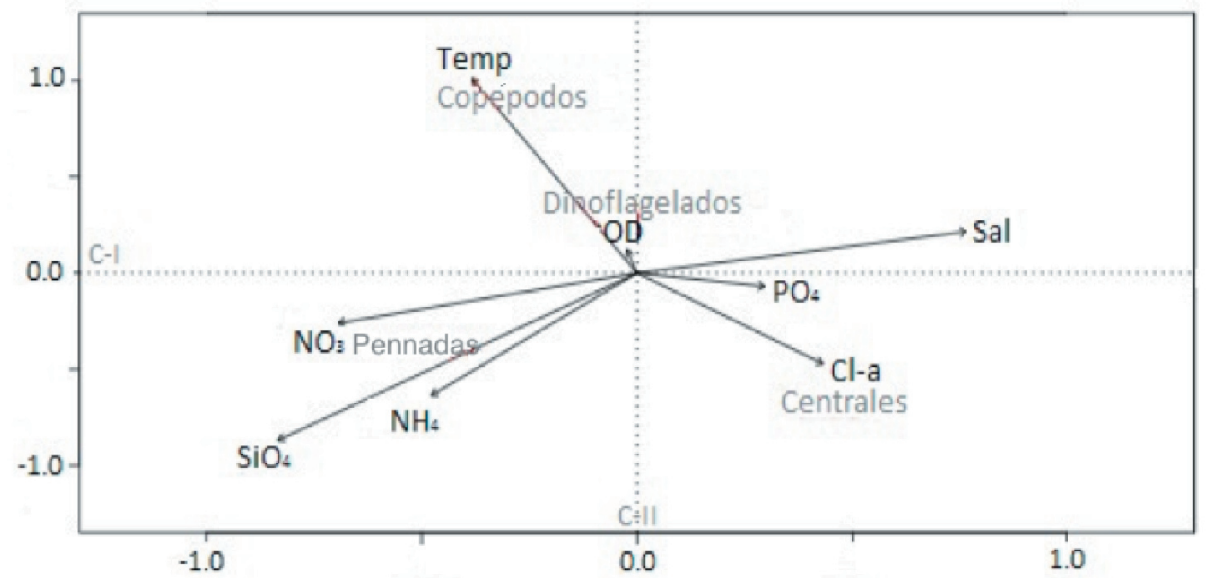

b)

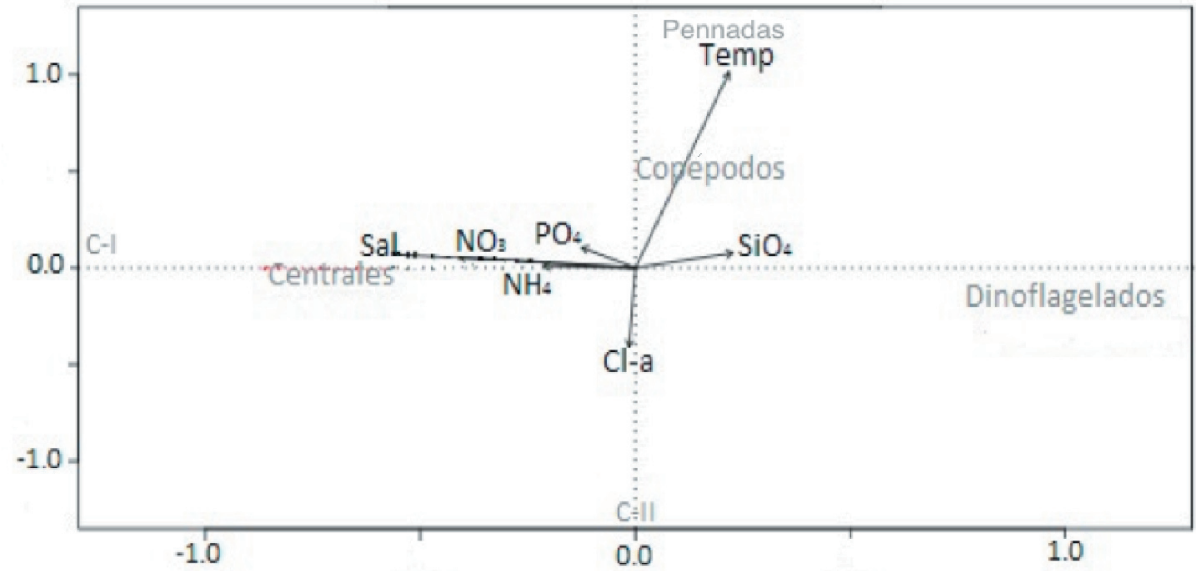

Figura 5. Proyecciones ortogonales del ACC (ejes canónicos C-I y C-II) entre los tres grupos de fitoplancton (diatomeas centrales y pennadas, y dinoflagelados) y los copépodos, y las variables hidroquímicas en a) Dzilam de Bravo y b) Cabo Catoche. Temp: temperatura, Sal.: salinidad, O.D.: oxígeno disuelto.

Figure 5. Ortothogonal proyections of the Corresponded Canonical Analysis (canonical axes C-I and C-II) among the three phytoplanktonic groups (centric and pennate Diatoms, and Dinoflagellates) and Copepods, and the hydrochemical variables in a) Dzilam de Bravo and b) Cabo Catoche. Temp: temperature, Sal.: salinity, O.D.: dissolved oxygen.

Se identificaron 15 grupos taxonómicos de zooplancton en Cabo Catoche durante los tres muestreos. El grupo dominante fue el de los copépodos (92.1\%). El 6.8\% restante se repartió entre los taxa Lamellibranchia (Bivalvia), Gastropoda, Sergestidae, Appendicularia, Echinodermata y Chaetognatha (Figura 4b).

El análisis de correspondencia (ACC) mostró asociación de los grupos de fitoplancton y los copépodos con las variables ambientales. En el eje canónico I (eigenvalue: 0.7413, C-I: $85.67 \%$ ), las diatomeas centrales se asociaron con la salinidad, nitratos, amonio y fosfatos, mientras que los dinoflagelados lo hicieron con los silicatos. Al eje II (eigenvalue: 0.1225, C-II: $14.15 \%$ ) se asociaron la temperatura junto con las diatomeas pennadas y copépodos, y la cl-a de manera inversa (Figura $5 b$ ).

Finalmente, se analizaron las variables hidroquímicas y planctónicas, y se las comparó durante los meses de intensificación de las
DAS (noviembre) y surgencia costera (agosto) según estudios previos (Merino 1992; 1997; Reyes-Mendoza et al. 2015). Es evidente que la intensificación de los fenómenos en ambos sitios ocasiona mayores concentraciones de nutrientes, con una mayor entrada de nitrógeno, ocasionando una alta relación $\mathrm{N}$ : P. Asimismo, todas las variables planctónicas analizadas resultaron ser significativamente diferentes $(\mathrm{P}<0.1)$, con excepción de la biomasa de zooplancton (Tabla 2).

\section{Discusión}

Los cambios temporales y espaciales en las variables hidroquímicas y planctónicas en los sitios representativos de influencia de los fenómenos oceanográficos, Dzilam de Bravo para las DAS y Cabo Catoche para la surgencia costera, sugieren que dichos fenómenos cumplen un papel importante en la estructura y en la relación entre los componentes de la comunidad de plancton costero en la PY. 
Las variaciones en los gradientes de temperatura y salinidad en Dzilam de Bravo y Cabo Catoche se relacionan con oscilaciones intra-anuales entre las fases de relajación e intensificación de las DAS y la surgencia costera. El comportamiento opuesto, tanto espacial como temporal, entre temperatura (mayor) y salinidad (menor) en Dzilam es resultado del profuso aporte de agua subterránea asociado a la recarga del sistema acuífero durante la temporada de lluvias (junio-octubre), proveyendo una masa de agua de baja salinidad y relativamente más cálida que la masa de agua costera (Herrera-Silveira 1993; Kantun-Manzano 2011; Vera et al. 2012). Por otro lado, a pesar de que temporalmente en Cabo Catoche no se registraron diferencias significativas en cuanto a la temperatura, la tendencia espacial decreciente de esta variable en función de la distancia a la costa sugiere una influencia del evento de surgencia en dicho sitio. Eso es consistente con lo reportado en estudios previos (Merino 1992; 1997; Reyes-Mendoza et al. 2015). Mientras que la intensificación de las DAS en noviembre genera para el área de Dzilam una masa de agua superficial más cálida $\left(>28^{\circ} \mathrm{C}\right)$ acotada a las inmediaciones de la costa (Enríquez et al. 2013), la surgencia costera ocasiona el arribo de una masa de agua superficial fría $\left(\leq 25^{\circ} \mathrm{C}\right)$, con salinidades cercanas a 36.5 ups en la región de Cabo Catoche, que se extiende mar adentro al menos $\sim 10 \mathrm{~km}$ de la costa.

Las mayores concentraciones de nitratos y silicatos registradas a lo largo de la transecta en estaciones adyacentes a la costa (estaciones 1 a 5) sugieren una influencia marcada de las DAS confinada a esta zona, ya que las descargas puntuales están distribuidas dentro de los primeros 50-500 $\mathrm{m}$ de la franja costera (Kantun-Manzano 2011). Temporalmente, la estacionalidad de las DAS se evidencia en concentraciones más elevadas (aunque no significativas) de nitratos y amonio en noviembre, cuando el sistema acuífero ha sido recargado como resultado de la temporada de lluvias que le antecede (Vera et al. 2012). En contraste, una alta concentración media de nitratos registrada en agosto en Cabo Catoche es consistente con la intensificación de la surgencia en este periodo del año (Merino 1997).

La relación positiva entre cl-a y densidad de diatomeas centrales hallada en el sitio de influencia de las DAS, conjuntamente con la relación negativa de ambos parámetros con el grupo de dinoflagelados (análisis multivariado ACC), indicarían que el incremento de la biomasa fitoplanctónica está explicado principalmente por el aumento de diatomeas dentro de la comunidad. Esta relación inversa entre densidad de diatomeas y de dinoflagelados podría estar reflejando diferentes estrategias de adaptación entre grupos de fitoplancton que habitan ambientes impactados por las DAS. Mientras que las diatomeas son especies perennes, caracterizadas por floraciones de alta diversidad y cosmopolitas en lo que se refiere al hábitat, los dinoflagelados son especies anuales, de floraciones monoespecíficas, ecofisiológicamente variables y especialistas en el hábitat que ocupan (Smayda 2002). Además, en Dzilam de Bravo, Álvarez-Góngora et al. (2012) asociaron las bajas salinidades y altas concentraciones de silicatos en la columna de agua influenciada por descargas de agua subterránea, con diatomeas de gran tamaño representativas de la región $(>30 \mu \mathrm{m})$. Por otro lado, los cambios opuestos de densidad de los grupos de fitoplancton (diatomeas centrales, pennadas y dinoflagelados) y los copépodos sugieren que la comunidad de fitoplancton podría estar siendo controlada mediante un mecanismo top-down. Los copépodos aumentaron gradualmente su densidad de junio a noviembre, mientras que los tres grupos fitoplanctónicos analizados registraron su menor densidad media durante el último mes de dicho período. Los cambios en las densidades de fitoplancton y copépodos sugieren una relación presa-depredador, la cual parecería acentuarse cuando las DAS son más intensas. Pese a que se muestreó sólo en una fecha de mayor intensidad del fenómeno, se sugiere que el aumento en la intensidad de turbulencia y los efectos de mezcla ocasionados por las DAS podrían haber incrementado la tasa de pastoreo ya que las posibilidades de encuentro del fitoplancton y el zooplancton aumentan si la columna de agua se encuentra en constante movimiento (Pécseli et al. 2014). Es importante mencionar que la fracción de zooplancton menor a 300 $\mu \mathrm{m}$ (microzooplancton) pudo haber tenido un impacto en el fitoplancton, pero no fue tenida en cuenta debido la técnica de muestreo utilizada. Sin embargo, al ser los copépodos el grupo dominante de zooplancton en la PY (Álvarez-Cadena et al. 2007), asumimos que dicho taxón es representativo de la comunidad.

En el sitio impactado por la surgencia costera, Cabo Catoche, la biomasa fitoplanctónica (cla) aumentó de forma significativa en la fecha 
de muestreo de mayor intensificación del fenómeno oceanográfico (agosto). Además, la densidad de diatomeas centrales aumentó considerablemente, lo que evidencia una dominancia clara en la comunidad. La relación negativa entre este grupo fitoplanctónico y los silicatos (análisis multivariado ACC) podría deberse a un consumo elevado de silicatos por parte de las diatomeas en los días precedentes al muestreo, en concordancia con lo reportado por Armbrecht et al. (2014). La dominancia de diatomeas en ecosistemas de surgencia se encuentra bien documentada. Wilkerson et al. (2006) han reportado que las diatomeas predominan en las primeras etapas del evento de surgencia en la costa Norte de California, lo cual se debería a las adaptaciones fisiológicas (e.g., alta tasa de consumo de nitratos en etapas tempranas del evento) que presentan dichos organismos (Lomas and Gilbert 1999). De la misma manera, Ochoa et al. (2010) y Pérez et al. (2010) describen cambios en la comunidad de fitoplancton en el ecosistema de surgencia de Humbolt e Iberia, respectivamente, y sugieren una alternancia en la dominancia por diatomeas en periodos de intensificación y dinoflagelados en periodos de relajación del fenómeno. Los dinoflagelados son organismos que están mejor adaptados a utilizar altas concentraciones de nutrientes en condiciones de baja turbulencia (Sellner et al. 2001), por lo que el ecosistema de surgencia sería un ambiente poco propicio para que dicho grupo alcance su desarrollo máximo.

La clara dominancia de diatomeas centrales en el mes de intensificación (agosto) de la surgencia de Cabo Catoche y de dinoflagelados en el mes de relajación (noviembre) revela que la estructura de la comunidad de fitoplancton varió notablemente, y respondió a las condiciones locales de concentración de nutrientes impuestas por el evento de surgencia. A pesar de que el fosfato sería limitante en todas las fechas de muestreo (i.e., relación N:P fue mayor en el mes de intensificación de la surgencia), se sugiere que la alta tasa de consumo de nitratos por parte de las diatomeas, característica en las primeras etapas de la surgencia (Lomas and Gilbert 1999), explicaría la dominancia de este grupo. El incremento significativo de densidad de las diatomeas centrales (dos órdenes de magnitud mayor que el resto de los grupos de fitoplancton estudiados), coincidente con concentraciones bajas de silicatos en el mes de agosto, indicaría un consumo importante de este nutriente antes del muestreo, sugiriendo que las diatomeas observadas son principalmente consecuencia de dicho consumo. Sin embargo, también es posible que parte de las diatomeas centrales encontradas en las capas superficiales durante la intensificación de la surgencia hayan sido transportadas por la turbulencia generada por el fenómeno desde capas más profundas. De esta manera, la variación en las condiciones hidroquímicas locales generadas por la surgencia costera ocasionó respuestas diferenciales en los grupos de fitoplancton, resultando en cambios importantes en la estructura de la comunidad.

De acuerdo a lo expuesto anteriormente, nuestra hipótesis de trabajo, que sugería un control bottom-up de la comunidad de fitoplancton durante intensificación de los fenómenos en ambos sitios, sólo podría sugerirse como una explicación plausible al comportamiento del fitoplancton en el sitio influenciado por la surgencia costera. Podrían existir varios factores que estén contribuyendo a la diferente respuesta de la comunidad de fitoplancton y zooplancton en los sitios influenciados por las DAS y la surgencia costera, y que probablemente sean emergentes en cada uno de ellos: variaciones en la intensidad de luz al mezclarse dos masas de agua de diferente densidad, adaptaciones al choque osmótico de los copépodos depredadores, o de ciertos grupos de fitoplancton al choque térmico por agua fría (surgencia costera), condiciones de turbulencia más propicias para el encuentro de los copépodos con el fitoplancton (Pécseli et al. 2014; Pino-Pinuer et al. 2014).

En conclusión, en el sitio impactado por las DAS la respuesta de la comunidad de fitoplancton y zooplancton reflejó un aumento de la densidad de copépodos y una disminución de diatomeas centrales, pennadas y dinoflagelados, lo que sugiere un cierto control de la comunidad de fitoplancton por parte de dicho grupo zooplanctónico. Por otro lado, en el sitio influenciado por la surgencia costera se observó un comportamiento distintivo en la comunidad de fitoplancton: dominancia de diatomeas centrales en agosto, correspondiente al periodo de intensificación, y de dinoflagelados en noviembre, correspondiente al periodo de relajación de la surgencia. Esto sugiere que la estructura de la comunidad de fitoplancton en Cabo Catoche estaría controlada por las condiciones hidroquímicas locales. Lo anterior indica que las DAS y la surgencia costera afectan de manera diferente la hidroquímica 
de la región costera de la PY e impactan en los niveles tróficos bajos, lo que genera respuestas diferenciales y cambios en la estructura de la comunidad de fitoplancton y en la relación entre sus componentes. Futuros estudios sobre la dinámica y estructura de la comunidad de fitoplancton y sus posibles controles bottomup y top-down en la PY podrían ser evaluados mediante trabajos con un diseño de muestreo temporal más intenso, relacionado con los tiempos generacionales del fitoplancton y zooplancton. En esta región del Golfo de México, estos dos fenómenos podrían estar teniendo influencia significativa en la biodiversidad planctónica, pero se desconoce su impacto a nivel de las funciones del ecosistema.
Agradecimientos. A los integrantes del laboratorio de productividad primaria y plancton del CINVESTAV-IPN Unidad Mérida, en especial a Iliana Osorio, Javier Ramírez, Fany Merino, Uriel Ordoñez y Margarita Ornelas. A Oscar Reyes-Mendoza, Natalí Cárdenas-Palomo y Emanuel MimilaHerrera por sus consejos y colaboración en los muestreos. Al Dr. Fernando R. Momo por sus siempre muy pertinentes comentarios.

Este estudio fue financiado parcialmente por el programa regular de becas académicas de la OEA (Argentina) y por el proyecto FOMIX Conacyt-Yucatán Ref: 108897, convocatoria M0023-2008-06 y FOPREDEN-CONABIOCINVESTAV.

\section{REFERENCIAS}

Álvarez-Cadena, J. N., U. Ordóñez-López, D. Valdés-Lozano, A. R. Almaral-Mendívil, and A. Uicab-Sabido. 2007. Estudio anual del zooplancton: composición, abundancia, biomasa e hidrología del Norte de Quintana Roo, mar Caribe de México. Revista mexicana de biodiversidad 78:421-430.

Álvarez-Góngora, C., and J. A. Herrera-Silveira. 2006. Variations of phytoplankton community structure related to water quality trends in a tropical karstic coastal zone. Marine Pollution Bulletin 52:48-60.

Álvarez-Góngora, C., M. A. Liceaga-Correa, and J. A. Herrera-Silveira. 2012. Variaciones estacionales de la estructura comunitaria del fitoplancton en zonas de descarga de agua subterránea en la costa Norte de la Península de Yucatán. Revista de Biología Tropical 60:157-172.

Armbrecht, L. H., M. Roughan, V. Rossi, A. Schaeffer, P. L. Davies, A. M. Waite, and L. K. Armand. 2014. Phytoplankton composition under contrasting oceanographic conditions: Upwelling and downwelling (Eastern Australia). Continental Shelf Research 75:54-67.

Boltovskoy, D. V. 1999. South Atlantic Zooplankton. Backhuys Publishers, Leiden, Holanda.

Cárdenas'Palomo, N., J. A. Herrera®Silveira, I. VelázquezロAbunader, O. Reyes, and U. Ordoñez. 2015. Distribution and feeding habitat characterization of whale sharks Rhincodon typus in a protected area in the north Caribbean Sea. Journal of fish biology 86:668-686.

Cloern, J. E., S. Q. Foster, and A. E. Kleckner. 2014. Phytoplankton primary production in the world's estuarine-coastal ecosystems. Biogeosciences 11:2477-2501.

Connell, J. H. 1978. Diversity in tropical rain forests and coral reefs. Science 199:1302-1310.

Corredor, J. E. 1979. Phytoplankton response to low-level nutrient enrichment through upwelling in the Columbian Caribbean Basin. Deep-Sea Research 26:731-741.

Di Rienzo, J. A., F. Casanoves, M. G. Balzarini, L. González, M. Tablada, and C. W. Robledo. 2014. InfoStat, versión 2014, Grupo InfoStat, FCA, Universidad Nacional de Córdoba, Argentina.

Enríquez, C., and I. Marino-Tapia. 2014. Mechanisms driving a coastal dynamic upwelling. In Proceedings of the 17th Physics of Estuaries and Coastal Seas (PECS) Conference.

Enríquez, C., I. Mariño-Tapia, G. Jeronimo, and L. Capurro-Filograso. 2013. Thermohaline processes in a tropical coastal zone. Continental Shelf Research 69:101-109.

Estrada, M., and D. Blasco. 1985. Phytoplankton Assemblages in Coastal Upwelling Areas. Pages 379-402 in C. Bas, R. Margalef and P. Rubias (eds.). International Symposium of Upwelling of W Africa, vol. 1. Instituto de Investigaciones Pesqueras, Barcelona.

Flöder, S., and U. Sommer. 1999. Diversity in planktonic communities: an experimental test of the intermediate disturbance hypothesis. Limnology and Oceanography 44:1114-1119.

Gobler, C. J., and G. E. Boneillo. 2003. Impacts of anthropogenically influenced groundwater seepage on water chemistry and phytoplankton dynamics within a coastal marine system. Marine Ecology Progress Series 255:101-114.

Hasle, G. R. 1978. The inverted-microscope methods Pages 88-96 in A. Sournia (ed.). Phytoplankton Manual. UNESCO, Paris, Francia.

Herrera-Silveira, J. A. 1993. Ecología de los productores primarios en la laguna de Celestún, México. Patrones de variación espacial y temporal. Tesis doctoral. Universidad de Barcelona, Barcelona. España. Pp. 233.

Herrera-Silveira, J. A. 2006. Lagunas costeras de Yucatán (SE, México): Investigación, diagnóstico y manejo. Ecotrópicos 19:94-108.

Herrera-Silveira, J. A., F. Comín, N. Aranda-Cirerol, L. Troccoli, and L. Capurro. 2004. Coastal water quality assessment in the Yucatan Peninsula: management and implications. Ocean and Coastal Management 47:625-639.

Huang, B., W. Xiang, X. Zeng, K. P. Chiang, H. Tian, J. Hu, W. Lan, and H. Hong. 2011. Phytoplankton growth and 
microzooplankton grazing in a subtropical coastal upwelling system in the Taiwan Strait. Continental Shelf Research 31:S48-S56

Hinojosa-Álvarez, S., F. Galván-Magaña, and X. Chiappa. 2012. Ecología Trófica de la Manta Gigante en el Caribe Mexicano. Un enfoque diferente al estudio de los hábitos alimentarios. Editorial Académica Española, España.

Jeffrey, S. W., and G. F. Humphrey. 1975. New spectrophotometric equations for determining chlorophylls a, b in higher plants, algae and natural phytoplankton. Biochemical Physiollogy Pflanzen 167:191-194.

Jiménez, R. 1981. Composition and distribution of phytoplankton in the upwelling systems of the Galapagos Islands. Pp. 327-338 in F. A. Richards (ed.). Coastal upwelling, Coastal Estuarine Sci. Vol. 1. AGU.

Johannes, R. E. 1980. The ecological significance of the submarine discharge of groundwater. Marine Ecology Progress Series 3:365-373.

Kantun-Manzano, C. A. 2011. Aporte de nutrients de las descargas submarinas del acuífero en Dzilam de Bravo, Yucatán. Tesis de maestría, Centro de Investigación y de Estudios Avanzados del Instituto Politécnico Nacional (CINVESTAV-IPN), Unidad Mérida, Yucatán. México. Pp. 220.

Legendre, L., and F. Rassoulzadegan. 1995. Plankton and nutrient dynamics in marine waters. Ophelia 41:153-172.

Lomas, M. W., and P. M. Gilbert. 1999. Temperature regulation of nitrate uptake: a novel hypothesis about nitrate uptake and reduction in cool-water diatoms. Limnology and Oceanography 44:556-572.

Margalef, R. 1967. Ritmos, fluctuaciones y sucesión. Pp. 454-492 en Margalef et al. (eds.). Ecología Marina. Fundación La Salle Cienc. Nat., Caracas, Venezuela.

Merino, I. M. 1992. Afloramiento en la plataforma de Yucatán: estructura y fertilización. Tesis doctoral. Universidad Nacional Autónoma de México, México, D.F. Pp. 276.

Merino, I. M. 1997. Upwelling on the Yucatan Shelf: hydrografic evidence. Journal of Marine Systems 13:101-121.

Ochoa, J., H. Sheinbaum, A. Badan, J. Candela, and D. Wilson. 2001. Geostrophy via potential vorticity inversion in the Yucatan Channel. Journal of Marine Research 59:725-747.

Ochoa, N., M. H. Taylor, S. Purca, and E. Ramos. 2010. Intra- and interanual variability of nearshore phytoplankton biovolume and community changes in the northern Humboldt Current system. Journal of Plankton Research 32: 843-855.

Odum, E. P., and G. Warrett. 2006. Fundamentos de ecología. 5ta edición. Thomson, Nueva York, USA.

Oksanen, J., F. G. Blanchet, R. Kindt, P. Legendre, P. R. Minchin, R. B. O'Hara, M. Henry, H. Stevens, and M. J. Oksanen. 2013. Package 'vegan'. Community ecology package, version 2(9).

Omori, M., and T. Ikeda. 1984. Methods in Marine Zooplankton Ecology. J. Wiley and Sons, Nueva York, USA.

Ordóñez-López, U., and M. Ornelas-Roa. 2003. Variaciones de la comunidad de copépodos plánticos en el gradiente estuarino-costero de Celestún, Yucatán, México. Hidrobiológica 13:231-238.

Padisák, J., C. S. Reynolds, and U. Sommer. (eds.). 1993. Intermediate Disturbance Hypothesis in Phytoplankton Ecology: Proceedings of the 8th Workshop of the International Association of Phytoplankton Taxonomy and Ecology held in Baja (Hungary), 5-15 July 1991 (Vol. 81). Springer Science and Business Media.

Parsons, T., Y. Maita, and C. Lally. 1984. A Manual of Chemical and Biological Methods of Seawater Analysis. Pergamon Press, Reino Unido.

Pécseli, H. L., J. K. Trulsen, and Ø. Fiksen. 2014. Predator-prey encounter and capture rates in turbulent environments. Limnology and Oceanography: Fluids and Environments 4:85-105.

Pérez, F. F., X. A. Padín, Y. Pazos, M. Golcito, M. Cabanas, P. C. Pardo, M. D. Doval, and L. Farina-Bustos. 2010. Plankton response to weakening of the Iberian coastal upwelling. Global Change Biology 16:1258-1267.

Peterson, T. D., H. N. J. Toews, C. L. K. Robinson, and P. J. Harrison. 2007. Nutrient and phytoplankton dynamics in the Queen Charlotte Islands (Canada) during the summer upwelling seasons of 2001-2002. Journal of Plankton Research 29:219-239.

Pino-Pinuer, P., R. Escribano, P. Hidalgo, R. Riquelme-Bugueño, and W. Schneider. 2014. Copepod community response to variable upwelling conditions off central-southern Chile during 2002-2004 and 2010-2012. Marine Ecology Progress Series 515:83-95.

Reyes-Mendoza, O. F. 2010. Caracterización espacial y temporal de la columna de agua en la Reserva de la Biosfera del Tiburón Ballena (Rhincodon typus) ubicada al noreste de la Península de Yucatán. Tesis de maestría. Centro de Investigación y de Estudios Avanzados del Instituto Politécnico Nacional (CINVESTAV-IPN), Unidad Mérida, Yucatán, México. Pp. 200.

Reyes-Mendoza, O., I. Mariño-Tapia, J. A. Herrera-Silveira, G. Ruiz-Martínez, C. Enríquez, and J. L. Largier. 2015. The effects of wind on upwelling off Cabo Catoche. Journal of Coastal Research 32:638-650.

Reynolds, C. S. 1993. Scales of disturbance and their role in plankton ecology. Hydrobiologia 249:157-171.

Round, F. E., R. M. Crawford, and D. G. Mann. 1990. The diatoms, biology and morphology of the genera. Cambridge University, Cambridge, Reino Unido.

Sellner, K. G., S. G. Sellner, R. V. Lacouture, and R. E. Magnien. 2001. Excessive nutrients select for dinoflagellates in the stratified Patapsco River estuary: Margalef reigns. Marine Ecology Progress Series 220:93-102.

Smayda, T. J. 2002. Adaptative ecology, growth strategies and the global bloom expanssion of dinoflagellates. Journal of Oceanography 58:281-294.

Sournia, A. 1986. Atlas du phytoplankton marin: Introduction, Cyanophycées, Dictychophycées, Dinophycées et Raphidophycées. Centre National de la Recherche Scientifique, Paris, Francia. 
Stolte, W., T. McCollin, A. A. Noordeloos, and R. Riegman. 1994. Effect of nitrogen source on the size distribution within marine phytoplankton populations. Journal of experimental marine Biology and Ecology 184:83-97.

Suárez-Morales, E., and R. Gasca. 2000. The planktonic copepod community at Mahahual reef, Western Caribbean. Bulletin of Marine Science 66:255-267.

ter Braak, C. J., and P. F. Verdonschot. 1995. Canonical correspondence analysis and related multivariate methods in aquatic ecology. Aquatic sciences 57:255-289.

Troccoli-Ghinaglia, L., J. A. Herrera-Silveira, and F. Comín. 2004. Structural variations of phytoplankton in the coastal seas of Yucatan, México. Hydrobiologia 519:85-102.

Troccoli-Ghinaglia, L., J. A. Herrera-Silveira, F. A. Comín, and J. R. Díaz-Ramos. 2010. Phytoplankton community variations in tropical coastal area affected where submarine groundwater occurs. Continental Shelf Research 30: 2082-2091.

Valle-Levinson, A., I. Mariño-Tapia, C. Enriquez, and A. F. Waterhouse. 2011. Tidal variability of salinity and velocity fields related to intense point-source submarine groundwater discharges into the coastal ocean. Limnology and Oceanography 56:1213-1224.

Vera, I., I. Mariño-Tapia, and C. Enríquez. 2012. Effects of drought and subtidal sea level variability on salt intrusion in a coastal karst aquifer. Marine Freshwater Research 63:485-493.

Wilkerson, F. P., R. C. Dugdale, A. Marchi, V. Hogue, and A. M. Lassiter. 2006. The phyplankton bloom response to wind events and upwelled nutrients during the COOP-WEST study. Deep Sea Research II 53:3023-3048. 